\title{
Serum and Urine Uric Acid as a Biomarker in Osteoarthritis
}

\author{
Jae-Bum Jun, M.D., Ph.D. \\ Department of Rheumatology, Hanyang University Hospital for Rheumatic Diseases, Seoul, Korea
}

It is common practice to use biomarkers not only in basic and clinical research but also in clinical practice, and currently there is no doubt that biomarkers are positioned as primary endpoints in many clinical trials [1]. Biomarker is a compound word for biological markers, which World Health Organization defines as "almost any measurements reflecting an interaction between a biological system and a potential hazard, which may be chemical, physical, or biological" [2].

The biomarkers in osteoarthritis (OA) can be classified as dry biomarkers and web soluble biomarkers [3]. Dry biomarkers include imaging parameters (radiograph, magnetic resonance imaging [MRI], and ultrasonography), questionnaires, and data from visual analog scales. Wet soluble biomarkers include genetic (DNA, RNA) and biochemical molecules.

The reason why a biomarker is needed in OA is to diagnose early disease, identify patients at risk of progression, and test new interventions [4]. To date, research on biomarkers has been very active in early diagnosis of $\mathrm{OA}$, prediction of progression, and treatment response [3].

Uric acid (UA) is an end-product of purine metabolism and is recognized as a biomarker for several cardiovascular diseases, renal diseases, and metabolic syndromes, as well as gout $[5,6]$. The relationship between uric acid, hyperuricemia, or gout and OA has been suggested by several researchers [7-11].

In 2017, Krasnokutsky et al. [12] reported that baseline serum UA and the progression of OA were correlated. Baseline serum UA was measured in 88 patients with knee OA using the Kellgren-Lawrence (K-L) grade $\geq 1$ symptomatic index knee. Joint space width and K-L grade were measured in knee X-rays at 0 and 24 months.
Baseline knee MRI was acquired from 27 subjects to measure synovial volume (SV). First, serum UA did not correlate with baseline OA severity, joint space width, or $\mathrm{K}-\mathrm{L}$ grades, as in previous studies. Univariate analysis showed serum UA was significantly associated with baseline SV measured by MRI, but was not associated with age, gender, and body mass index (BMI) in multivariate analysis. Most importantly, serum UA was significantly associated with $\mathrm{OA}$ progression as measured by median joint space narrowing for 24 months $(r=0.40, p<0.01)$, and was maintained even after adjusting for BMI, gender, and age $(\mathrm{r}=0.28, \mathrm{p}=0.01)$. When radiologic progression was divided into non-progressor, slow progressor, and fast progressor groups, there was a significant difference in serum UA between the fast progressor and non-progressor groups. ROC-AUC curves showed serum UA could predict knee OA progression after 24 months, and serum UA significantly predicted progressors from non-progressors. The authors said that this was the first longitudinal study to report that serum UA could act as a biomarker for OA progression.

In a recent retrospective study by Xiao et al. [13], the association between serum UA and MRI findings (synovitis and soft tissue swelling) was reported.

In the past issue of Journal of Rheumatic Diseases, Kim et al. [14] reported that serum and urine uric acid were associated with synovial inflammation and radiographic progression as measured by musculoskeletal ultrasound (MSUS). Serum and urine uric acid were measured in 42 patients with knee OA and 58 healthy controls, and the K-L grade and MSUS findings were compared among knee OA patients. First, the urine uric acid/creatinine ratio in OA patients was significantly higher in K-L grade III than

Received : February 17, 2020, Accepted : February 19, 2020

Corresponding to : Jae-Bum Jun (iD http://orcid.org/0000-0002-0208-0505

Department of Rheumatology, Hanyang University Hospital for Rheumatic Diseases, 222 Wangsimni-ro, Seongdong-gu, Seoul 04763, Korea. E-mail : junjb@hanyang.ac.kr 
in K-L grade II. In the association between serum UA and MSUS findings, serum UA was closely related to synovial hypertrophy, and serum UA and serum/urine UA ratio were higher in OA with synovial hypertrophy compared to OA without synovial hypertrophy. Finally, the multivariate analysis showed only urine UA had a negative association with knee OA (odds ratio $=0.974,95 \%$ confidence interval, $0.954 \sim 0.994, p=0.013)$. In conclusion, serum UA was closely associated with synovial inflammation, and urine uric acid/creatinine ratio, which reflect excretion of uric acid per day, were associated with cartilage damage and radiographic OA progression. This was the first study to report the association between UA in urine as well as serum and the progression of OA.

First, uric acid is considered an excellent biomarker in that it is an affordable, easily measurable test at any level of medical institution. However, it should be taken into account that UA levels can be greatly affected by the subject's dietary conditions, health conditions, and the timing of measurement. OA is especially common in old age and is associated with comorbidities, such as many cardiovascular and kidney diseases, making polypharmacy a likely confounder $[15,16]$. Therefore, the fact that UA measured in OA patients can be greatly affected by the health condition of the patient, the medication taken may limit its value as a biomarker. Kim et al. [14] have reported that urine uric acid is related to OA. The authors noted that spot urine $\mathrm{UA} /$ creatinine could replace 24-hour uric acid excretion as a biomarker, but the references cited were abstracts and the results of studies in gout patients [17]. Moriwaki et al. [18] found that early morning urine $\mathrm{UA} /$ creatinine was associated with 24-hour uric acid excretion, but this association was present only in gout patients and not in healthy controls. Therefore, the use of spot urine UA as a biomarker for OA seems to require further research. Rather, fractional excretion of UA might be an alternative [19]. In addition, considering that the period of recruitment for the subjects in Kim et al. [14] was one year, it should also be considered that the levels for serum UA and 24-hour primary UA vary from month to month as reported by Yu et al. [20]. It may be very prudent to conclude that the progression of knee OA can be predicted by measuring uric acid once in the blood or urine, which can be affected by these various variables and which may vary in measurement.

Second, in 2017 Krasnokutsky et al. [12] studied OA with symptoms in at least one knee that met clinical American College of Rheumatology criteria, and des- ignated knees with symptoms as index knees. A pain questionnaire, knee X-ray, and MRI were also performed. A study by Kim et al. [14] did not explain their criteria for the index knee. Therefore, it is not known which knee was evaluated by knee X-ray and MSUS. If knee X-ray and MSUS were performed on the most severe knee of both sides, it is necessary to explain how one uric acid level can be used to correlate the condition of the knee with or without $\mathrm{OA}$ as the biomarker for both knees at the same time. This is true of other previous studies.

Third, in explaining the association between UA and $\mathrm{OA}$, the secretion of inflammatory cytokines interleukin (IL) $-1 \beta$ and IL-18 along with uric acid activation of the NLRP3 inflammasome has been discussed. Denoble et al. [8] reported that UA in synovial fluid was associated with synovial IL-1 $\beta$ and IL-18 levels, and that synovial fluid UA was associated with knee OA severity. In 2013, UA, IL-1 $\beta$, IL-18, and caspase- 1 were measured in synovial fluids of several types of arthritis including OA [21]. At that time, the number of OA patients was small [11], but the concentrations of synovial fluid IL-1 $\beta$ and IL-18 were compatible with those of Denoble et al. [8]. However, the concentrations of IL-1 $\beta$ and IL-18 were significantly lower in OA synovial fluid than in other diseases. In addition, caspase-1 levels were significantly lower in $\mathrm{OA}$ synovial fluid. Given these results, it is questionable whether the role of IL- $1 \beta$ and IL-18 in activating the NLRP3 inflammasome by uric acid can be well explained in OA progression. This will need to be explained.

Although we use biomarkers as surrogate endpoints in clinical trials, biomarkers are different from clinical endpoints. As Kim et al. [14] pointed out in their limitations, it is necessary to conduct large-scale clinical studies to determine if serum or urine UA can serve as predictive or prognostic biomarkers for OA progression.

\section{CONFLICT OF INTEREST}

No potential conflict of interest relevant to this article was reported.

\section{REFERENCES}

1. Strimbu K, Tavel JA. What are biomarkers? Curr Opin HIV AIDS 2010;5:463-6.

2. International Programme on Chemical Safety. Biomarkers and risk assessment: concepts and principles [Internet]. Geneva: World Health Organization, c1993 [cited 2020]. Available from: http://www.inchem.org/documents/ehc/ 
ehc/ehc155.htm.

3. Mobasheri A. Osteoarthritis year 2012 in review: biomarkers. Osteoarthritis Cartilage 2012;20:1451-64.

4. Watt FE. Osteoarthritis biomarkers: year in review. Osteoarthritis Cartilage 2018;26:312-8.

5. Johnson RJ, Kang DH, Feig D, Kivlighn S, Kanellis J, Watanabe $\mathrm{S}$, et al. Is there a pathogenetic role for uric acid in hypertension and cardiovascular and renal disease? Hypertension 2003;41:1183-90.

6. Borges RL, Ribeiro AB, Zanella MT, Batista MC. Uric acid as a factor in the metabolic syndrome. Curr Hypertens Rep 2010;12:113-9.

7. Acheson RM, Collart AB. New Haven survey of joint diseases. XVII. Relationship between some systemic characteristics and osteoarthrosis in a general population. Ann Rheum Dis 1975;34:379-87.

8. Denoble AE, Huffman KM, Stabler TV, Kelly SJ, Hershfield MS, McDaniel GE, et al. Uric acid is a danger signal of increasing risk for osteoarthritis through inflammasome activation. Proc Natl Acad Sci U S A 2011;108:2088-93.

9. Ding X, Zeng C, Wei J, Li H, Yang T, Zhang Y, et al. The associations of serum uric acid level and hyperuricemia with knee osteoarthritis. Rheumatol Int 2016;36:567-73.

10. Ma CA, Leung YY. Exploring the link between uric acid and osteoarthritis. Front Med (Lausanne) 2017;4:225.

11. Neogi T, Krasnokutsky S, Pillinger MH. Urate and osteoarthritis: evidence for a reciprocal relationship. Joint Bone Spine 2019;86:576-82.

12. Krasnokutsky S, Oshinsky C, Attur M, Ma S, Zhou H, Zheng $\mathrm{F}$, et al. Serum urate levels predict joint space narrowing in non-gout patients with medial knee osteoarthritis. Arthritis Rheumatol 2017;69:1213-20.

13. Xiao L, Lin S, Zhan F. The association between serum uric acid level and changes of MRI findings in knee osteoarthritis: a retrospective study (A STROBE-compliant article). Medicine (Baltimore) 2019;98:e15819.

14. Kim SK, Jung UH, Choe JY. Clinical usefulness of uric acid as a biomarker for knee osteoarthritis: a comparative analysis with plain radiography and musculoskeletal ultrasound. J Rheum Dis 2020;27:51-60.

15. Hawker GA. Osteoarthritis is a serious disease. Clin Exp Rheumatol 2019;37 Suppl 120:3-6.

16. Swain S, Sarmanova A, Coupland C, Doherty M, Zhang W. Comorbidities in Osteoarthritis: A systematic review and meta-analysis of observational studies. Arthritis Care Res (Hoboken) 2019 Jun 17 [Epub]. DOI: 10.1002/acr.24008.

17. Choi S, Moon SJ, Kang EJ, Lee KH. Validity of random urinary uric acid-to-creatinine ratio for estimating 24-hour urine uric acid excretion in patients with gout. Ann Rheum Dis 2018;77(Suppl 2):664-5.

18. Moriwaki Y, Yamamoto T, Takahashi S, Yamakita J, Tsutsumi Z, Hada T. Spot urine uric acid to creatinine ratio used in the estimation of uric acid excretion in primary gout. J Rheumatol 2001;28:1306-10.

19. Kannangara DR, Ramasamy SN, Indraratna PL, Stocker SL, Graham GG, Jones G, et al. Fractional clearance of urate: validation of measurement in spot-urine samples in healthy subjects and gouty patients. Arthritis Res Ther 2012;14: R189.

20. Yu KH, Luo SF, Tsai WP, Huang YY. Intermittent elevation of serum urate and 24-hour urinary uric acid excretion. Rheumatology (Oxford) 2004;43:1541-5.

21. Son CN, Bang SY, Kim JH, Choi CB, Kim TH, Jun JB. Caspase-1 level in synovial fluid is high in patients with spondyloarthropathy but not in patients with gout. J Korean Med Sci 2013;28:1289-92. 